\title{
A SIMPLE PROOF OF HERMITE'S THEOREM ON THE ZEROS OF A POLYNOMIAL
}

\author{
by N. J. YOUNG
}

(Received 16 October, 1981)

In 1856 Hermite showed how to determine by purely rational operations the number of zeros of a given polynomial lying in a specified half plane [1]: one inspects the signature of a certain Hermitian form. This type of result is still of interest for practical applications, and several authors have provided alternatives to Hermite's original, highly computational proof (for example [2, 3]). Recently V. Pták and the author gave a simple matrix-theoretic proof and generalization of a class of Hermite-type theorems [4]. This class included the Schur-Cohn test for zeros in a circle, but not, to our regret, the original theorem of Hermite. The purpose of this note is to show that a slight modification of our method does indeed provide a simple proof of Hermite's theorem.

Hermite's test uses the Bezoutian $\operatorname{Bez}(f, g)$ of two polynomials $f$ and $g$ of degree $n$ (over $\mathbb{C}$ ): this is defined to be the $n \times n$ matrix $\left[c_{i j}\right]$, where

$$
\sum_{i, j=0}^{n-1} c_{i j} z^{i} w^{i}=\frac{f(z) g(w)-g(z) f(w)}{z-w}
$$

The present proof uses a matrix identity for the Bezoutian. It is a matter of straightforward computation that, if $f(z)=\sum_{0}^{n} a_{r} z^{r}$ and $g(z)=\sum_{0}^{n} b_{r} z^{r}$,

$$
\operatorname{Bez}(f, g)=\left[\sum_{k=0}^{i} b_{j-k} a_{i+1+k}-a_{i-k} b_{i+1+k}\right]_{i, j=0}^{n-1}
$$

This can also be written

$$
\begin{aligned}
& \operatorname{Bez}(f, g)=\left[\begin{array}{cccc}
a_{1} & a_{2} & \ldots & a_{n} \\
a_{2} & a_{3} & \ldots & 0 \\
. & . & \ldots & . \\
a_{n} & 0 & \ldots & 0
\end{array}\right]\left[\begin{array}{cccc}
b_{0} & b_{1} & \ldots & b_{n-1} \\
0 & b_{0} & \ldots & b_{n-2} \\
. & . & \ldots & . \\
0 & 0 & \ldots & b_{0}
\end{array}\right] \\
& -\left[\begin{array}{cccc}
b_{1} & b_{2} & \ldots & b_{n} \\
b_{2} & b_{3} & \cdots & 0 \\
\cdot & \cdot & \cdots & \cdot \\
b_{n} & 0 & \ldots & 0
\end{array}\right]\left[\begin{array}{cccc}
a_{0} & a_{1} & \ldots & a_{n-1} \\
0 & a_{0} & \cdots & a_{n-2} \\
\cdot & \cdot & \ldots & \cdot \\
0 & 0 & \ldots & a_{0}
\end{array}\right]
\end{aligned}
$$

Glasgow Math. J. 24 (1983) 125-128. 
or, if we introduce the matrices

$$
S=\left[\begin{array}{ccccc}
0 & 1 & 0 & \ldots & 0 \\
0 & 0 & 1 & \ldots & 0 \\
. & . & . & \ldots & . \\
0 & 0 & 0 & \ldots & 1 \\
0 & 0 & 0 & \ldots & 0
\end{array}\right], \quad J=\left[\begin{array}{ccccc}
0 & 0 & 0 & \ldots & 1 \\
0 & 0 & 0 & \ldots & 0 \\
. & . & . & \ldots & . \\
0 & 1 & 0 & \ldots & 0 \\
1 & 0 & 0 & \ldots & 0
\end{array}\right]
$$

of type $n \times n$,

$$
\operatorname{Bez}(f, g)=J f_{1}\left(S^{*}\right) g(S)-J g_{1}\left(S^{*}\right) f(S),
$$

where the star denotes the conjugate transpose and

$$
f_{1}(z)=z^{n} f(1 / z) \text {. }
$$

We shall also need the polynomials $f^{*}$ and $f_{0}$ defined by

$$
f^{*}(z)=f(\bar{z})^{-}, \quad f_{0}=\left(f_{1}\right)^{*} .
$$

HeRmite's theOREM. Let $f$ be a polynomial over $\mathbb{C}$. Suppose that the matrix

$$
B=-i \operatorname{Bez}\left(f, f^{*}\right)
$$

is non-singular. Then the number of + signs in the canonical form of the Hermitian form $x^{*} B x$ is equal to the number of zeros of $f$ with positive imaginary parts.

Proof. We can suppose $f$ monic. Let

Then

$$
f(z)=\left(z-\alpha_{1}\right)\left(z-\alpha_{2}\right) \ldots\left(z-\alpha_{n}\right) .
$$

$$
\begin{aligned}
f^{*}(z) & =\left(z-\bar{\alpha}_{1}\right)\left(z-\bar{\alpha}_{2}\right) \ldots\left(z-\bar{\alpha}_{n}\right), \\
f_{1}(z) & =\left(1-\alpha_{1} z\right)\left(1-\alpha_{2} z\right) \ldots\left(1-\alpha_{n} z\right), \\
f_{0}(z) & =\left(1-\bar{\alpha}_{1} z\right)\left(1-\bar{\alpha}_{2} z\right) \ldots\left(1-\bar{\alpha}_{n} z\right) .
\end{aligned}
$$

Introduce the $n \times n$ matrices

$$
E_{i}=S-\alpha_{i} I, \quad F_{i}=S-\bar{\alpha}_{i} I, \quad G_{i}=I-\alpha_{i} S, \quad H_{i}=I-\bar{\alpha}_{i} S .
$$

Then

$$
\begin{array}{rlrl}
f(S) & =E_{1} E_{2} \ldots E_{n}, & f^{*}(S) & =F_{1} F_{2} \ldots F_{n}, \\
f_{1}\left(S^{*}\right) & =H_{n}^{*} H_{n-1}^{*} \ldots H_{1}^{*}, \quad f_{0}\left(S^{*}\right)=G_{n}^{*} G_{n-1}^{*} \ldots G_{1}^{*} .
\end{array}
$$

From (1),

$$
\begin{aligned}
B & =-i \operatorname{Bez}\left(f, f^{*}\right) \\
& =-i J\left\{f_{1}\left(S^{*}\right) f^{*}(S)-f_{0}\left(S^{*}\right) f(S)\right\} \\
& =-i J\left\{H_{n}^{*} \ldots H_{1}^{*} F_{1} \ldots F_{n}-G_{n}^{*} \ldots G_{1}^{*} E_{1} \ldots E_{n}\right\} .
\end{aligned}
$$


We now appeal to an identity for the difference of two products. Consider the sum

$$
\sum_{k=1}^{n} H_{n}^{*} \ldots H_{k+1}^{*} G_{k-1}^{*} \ldots G_{1}^{*}\left(H_{k}^{*} F_{k}-G_{k}^{*} E_{k}\right) E_{1} \ldots E_{k-1} F_{k+1} \ldots F_{n} .
$$

(In the case $k=1$ we interpret $H_{n}^{*} \ldots H_{k+1}^{*} G_{k-1}^{*} \ldots G_{1}^{*}$ as $H_{n}^{*} \ldots H_{2}^{*}$, etc.) On writing out the sum we find that, in view of the fact that $H_{\mathrm{k}}^{*}$ commutes with $G_{i}^{*}$ and $E_{k}$ commutes with $E_{j}$, the second component of the $k$ th term cancels with the first component of the $(k+1)$ th term, $1 \leqslant k \leqslant n-1$, and the sum telescopes to

$$
H_{n}^{*} \ldots H_{1}^{*} F_{1} \ldots F_{n}-G_{n}^{*} \ldots G_{1}^{*} E_{1} \ldots E_{n},
$$

so that (2) can be written

$$
B=-i J \sum_{k=1}^{n} H_{n}^{*} \ldots H_{k+1}^{*} G_{k-1}^{*} \ldots G_{1}^{*}\left(H_{k}^{*} F_{k}-G_{k}^{*} E_{k}\right) E_{1} \ldots E_{k-1} F_{k+1} \ldots F_{n} .
$$

Now it is readily checked that

$$
\begin{aligned}
H_{i}^{*} F_{i}-G_{i}^{*} E_{j} & =\left(\alpha_{i}-\bar{\alpha}_{i}\right)\left(I-S^{*} S\right) \\
& =2 i\left(\operatorname{Im} \alpha_{j}\right) e e^{*},
\end{aligned}
$$

where $e^{*}=\left[\begin{array}{lll}1 & 0 & \ldots\end{array}\right]$. Thus

$$
B=-2 i J \sum_{k=1}^{n} i\left(\operatorname{Im} \alpha_{k}\right) H_{n}^{*} \ldots H_{k+1}^{*} G_{k-1}^{*} \ldots G_{1}^{*} e e^{*} E_{1} \ldots E_{k-1} F_{k+1} \ldots F_{n} .
$$

I claim that

$$
J H_{n}^{*} \ldots H_{k+1}^{*} G_{k-1}^{*} \ldots G_{1}^{*} e=F_{n}^{*} \ldots F_{k+1}^{*} E_{k-1}^{*} \ldots E_{1}^{*} e .
$$

To see this, note that if $h$ is any polynomial of degree $n-1$ and $h_{1}(z)=z^{n-1} h(1 / z)$ then $J h_{1}\left(S^{*}\right)$ and $h\left(S^{*}\right)$ have the same first column, which is to say that

$$
J h_{1}\left(S^{*}\right) e=h\left(S^{*}\right) e .
$$

On applying this observation with

$$
h(z)=\left(z-\alpha_{n}\right) \ldots\left(z-\alpha_{k+1}\right)\left(z-\bar{\alpha}_{k-1}\right) \ldots\left(z-\bar{\alpha}_{1}\right),
$$

one establishes the claim.

We now have

$$
\begin{aligned}
B & =2 \sum_{k=1}^{n}\left(\operatorname{Im} \alpha_{k}\right) F_{n}^{*} \ldots F_{k+1}^{*} E_{k-1}^{*} \ldots E_{1}^{*} e e^{*} E_{1} \ldots E_{k-1} F_{k+1} \ldots F_{n} \\
& =2 \sum_{k=1}^{n}\left(\operatorname{Im} \alpha_{k}\right) v_{k} v_{k}^{*}
\end{aligned}
$$

where

$$
v_{k}=F_{n}^{*} \ldots F_{k+1}^{*} E_{k-1}^{*} \ldots E_{1}^{*} e .
$$


This can be written

$$
B=V \operatorname{diag}\left\{\operatorname{Im} \alpha_{1}, \ldots, \operatorname{Im} \alpha_{n}\right\} V^{*}
$$

where

$$
V=\sqrt{ } 2\left[v_{1} \ldots v_{n}\right]
$$

If $B$ is non-singular then so is $V$ and hence $B$ is congruent to $\operatorname{diag}\left\{\operatorname{Im} \alpha_{1}, \ldots, \operatorname{Im} \alpha_{n}\right\}$. Hermite's assertion follows at once.

\section{REFERENCES}

1. C. Hermite, Sur le nombre des racines d'une équation algébrique comprises entre des limites données, J. Reine Angew. Math. 52 (1856), 39-51; translation in Internat. J. Control 26 (1977), 183-195.

2. P. C. Parks, Further comments on "A symmetric matrix formulation of the Hurwitz-Routh stability criterion”, IEEE Trans. Automat. Control 8 (1963), 270-271.

3. P. C. Parks, A new proof of Hermite's stability criterion and a generalization of Orlando's formula, Internat. J. Control 26 (1977), 197-206.

4. Vlastimil Pták and N. J. Young, A generalization of the zero location theorem of Schur and Cohn, IEEE Trans. Automat. Control 25 (1980), 978-980.

UNIVERSITY OF GLASGOW

DEPARTMENT OF MATHEMATICS

UNIVERSITY GARDENS

GLASGOW

G12 8QW 